\title{
Probabilistic Extension of the Backward/Forward Load Flow Analysis Method
}

\author{
Eduard Janecek and Daniel Georgiev
}

\begin{abstract}
The environmental need to curb distribution network losses and utilize renewable energy sources has created new challenges in estimation. High fidelity estimates are required even in the presence of significant uncertainty. Herein, we develop a new analytical probabilistic load flow method that, unlike existing analytical methods, is not based on a Taylor series approximation of the power equations. The method is exact for a set of distributions that includes the multivariate normal distribution. The method implementation is made scalable by casting all formulas into the framework of the popular backward/forward algorithm. The advantages of this approach are illustrated on a radial IEEE 32-bus test system. Significant improvements are observed in the presence of large power uncertainties and near the network power limits. Uniformly better estimation of power losses is achieved.
\end{abstract}

Index Terms-Load flow, power system analysis computing, power system modeling, uncertainty.

\section{NOMENCLATURE}

$\mathcal{N}=$

$\{1, \ldots, N\}$

$\mathcal{B} \in \mathcal{N} \times \mathcal{N} \quad$ Complete set of network branches.

$\mathcal{U}_{k} \subseteq \mathcal{N}$

Set of nodes upstream of node $k$.

$V_{k} \in \mathbb{C}$

Estimated voltage at node $k$.

$v_{0} \in \mathbb{R}$

Actual base voltage.

$I_{k} \in \mathbb{C}$

Estimated current through branch $k$.

$J_{k}=\mu_{k}+$

Estimated loop current at node $k$.

$\sigma_{k}^{T} W_{k} \in \mathbb{C}$

$S_{k}=P_{k}+$

Estimated load at node $k$.

$i Q_{k} \in \mathbb{C}$

$S_{0 k}=P_{0 k}+\quad$ Actual load at node $k$.

$i Q_{0 k} \in \mathbb{C}$

$\Delta S_{k} \in \mathbb{R}$

Estimated power loss across branch $k$.

$z_{k} \in \mathbb{C}$

Impedance of branch $k$.

$\rho_{R}, \rho_{I}, \rho_{R I}, \rho \in$ Correlation parameters of loop currents. $\mathbb{R}$

$R_{1 s} \in \mathbb{C}^{2 \times 2} \quad$ Covariance of $W_{k}, s \in\{H, T\}$.

Manuscript received November 30, 2010; revised December 09, 2010 and May 26, 2011; accepted August 22, 2011. Date of publication November 03, 2011; date of current version April 18, 2012. This work was supported in part by the grant TA ČR TA01020865. Paper no. TPWRS-00969-2010.

The authors are with the Department of Cybernetics in the College of Applied Sciences, University of West Bohemia, Pilsen 30000, Czech Republic (e-mail: janecek@kky.zcu.cz; georgiev@kky.zcu.cz).

Digital Object Identifier 10.1109/TPWRS.2011.2170443

$\begin{array}{ll}R_{2 s} \in \mathbb{C}^{2 \times 2} & \begin{array}{l}\text { Covariance of } W_{k} \text { with } W_{\ell}, \ell \neq k, s \in \\ \{H, T\} .\end{array} \\ \gamma \in \mathbb{R} & \text { Contemporaneity factor. } \\ x(k) \in \mathbb{C}^{5} & \text { Backward state at node } k . \\ y(k) \in \mathbb{C}^{8} & \text { Forward state at node } k . \\ U_{k} \in \mathbb{R}^{4} & \text { Vector of estimates at node } k \text { made up } \\ & \text { from the real and imaginary parts of } \\ & \left(J_{k}, V_{k}\right) . \\ \Sigma_{k} \in \mathbb{R}^{4 \times 4} & \text { Variance of } U_{k} . \\ \Gamma_{k s} \in \mathbb{C} & \text { Covariance of } J_{k} \text { with } V_{k}, s \in\{H, T\} . \\ \epsilon_{k} \in \mathbb{R}^{4} & \text { Power estimation error at node } k . \\ \mathcal{W}_{\mathcal{N}, V}, & \text { Wasserstein voltage and power loss } \\ \mathcal{W}_{\mathcal{N}, \Delta S} \in \mathbb{R} & \text { estimation errors. }\end{array}$

\section{INTRODUCTION}

$\mathbf{T}$ HE global effort to curb carbon emissions has created new challenges in distribution network design. Improvement of computational tools is integral to overcoming these challenges.

For instance, in order to meet renewable energy source utilization targets [1], better planning and source management tools need to be developed [1]. Renewable energy sources produce new uncertainties. Their power production is strongly correlated with environmental conditions, yet their regulation is limited as they are often distributed along low voltage networks. Current planning and management solutions, such as oversizing or production curtailing [1], [2], are not economically or ecologically efficient.

Load flow analysis is fundamental to both planning and management. When uncertainty is relevant, probabilistic load flow (PLF) analysis seems to be preferred due to the statistical language of the new European Union distribution network standards [2]. There are two general categories of PLF methods, i.e., simulation based and analytical [3], that each have distinct advantages, i.e., model accuracy and computational efficiency, respectively. Current analytical PLF methods efficiently compute output statistics using simple formulas based on various truncated Taylor series approximations of the power equations. Hence, they can be inaccurate when large deviations are common. Herein, we develop a new analytical PLF method that is not based on power equation approximation. 


\section{A. Literature Review}

Here we discuss the existing simulation based and analytical methods within the scope of our work. For a detailed review of PLF methods, we refer the reader to [3]. The section concludes with an overview of the deterministic backward/forward (b/f) method.

1) Simulation-Based Methods: Simulation-based methods are built on top of deterministic algorithms (such as the b/f algorithm or Newton's method). Monte Carlo methods are one example. A key advantage of Monte Carlo methods is their flexibility [4]. The most cited setback is the large number of runs required to accurately solve the PLF problem [3], [5]-[7]. The computational complexity becomes especially prohibitive for large networks because every node introduces a new load uncertainty that must be tested in combination with all others. For this reason, many higher level methods exist that combine Monte Carlo simulation with more efficient algorithms designed for specific scenarios [8].

2) Approximate Methods Based on Linearization: Probabilistic load flow analysis is complicated by the nonlinearity of the power equations [3]. Existing analytical methods are built on various approximations of these equations. Linearization is most often used, e.g., [9]-[12] and others cited in the recent review paper [3].

Linearization in PLF analysis was first introduced almost 40 years ago in [10], where convolution is used to study load flow in DC networks. Its extension to AC networks soon followed [11] and the general approach (see [13]) has remained the same since. First state means are estimated from the power means. Then the power equations are linearized around this point. Finally, the linearized model is used to directly compute the output moments from the power moments.

The basic method is fast and easy in principle. However, if the perturbations are large, the linearization point as well as the linearization are prone to significant errors [13]. Possible refinements either combine multiple linearizations or add modified quadratic terms. Multi-linearization is a higher order method that builds directly on the basic linearization method, e.g., Monte Carlo combined with linearization [8]. The quadratic PLF method retains some nonlinear structure by keeping the second order Taylor series terms, evaluated using the first order Taylor series approximation [14]. It yields only marginal improvements under special circumstances [3], perhaps because the method is still prone to the same linearization errors.

3) Approximate Methods Based on Point Estimates: Recently, an alternate approximation, based on a finite number of probability concentrations (see [15] for details), has been developed for PLF analysis [5], [7]. The so-called point estimate method (PEM) first solves the deterministic load flow problem at several sample points and then weighs them to estimate the output moments. A third order truncated Taylor series is used to derive the point locations and their weights.

The PEM method can outperform linearization methods in accuracy and Monte Carlo methods in computational complexity. For third order polynomial power equations, the method can compute the expectation values exactly given limited cross correlation between the power inputs [7], [16]. Moreover, the number of sample points required is proportional to the number of inputs. So the computational complexity of the underlying deterministic method is increased by only a single polynomial degree.

4) Backward/Forward LF Method: The b/f method is applicable for LF analysis of radial and weakly meshed networks [17], [18]. It has been developed for single-phase, three-phase, and four-wire systems [17], [19], [20]. It is efficient as no composite system model is required [21]. It is also modular as nodes simply update and exchange their local information with known neighbors. For these reasons, the method is often preferred for large systems and applications requiring repeated network estimation [18], [22], [23].

The general algorithm [17] for radial networks gets its name from its repeated backward and forward sweeps. In the backward sweep, estimates of either currents or power flows are summed and passed on from the leaf nodes to the root node. In the forward sweep, results of the backward sweep are used to estimate nodal voltages and loads. The sweeps are repeated until the estimated inputs are in sufficient agreement with the actual inputs. If the network is meshed, break points are defined that decompose it into several radial networks. Auxiliary currents are then injected at the breakpoints to compensate for the missing branches.

An alternative approach is to centralize the computation using a gradient descent scheme, e.g., the Newton-Rhapson method. This usually involves composing a full network model and inverting a gradient matrix of the full power flow equation system to find the descent direction. This may significantly increase the initialization time and the iteration time [24]. Moreover, the general methodology is more complex [24] and, by definition, less modular.

Nonetheless, a slow convergence rate can be a weakness of the b/f method [25]. A closer look reveals that the algorithm is a heuristic distributed polynomial solver, where each node updates its customer current with no regard for the updates at other nodes. It has been shown that for heavily loaded systems, the centralized approach may converge in fewer iterations with an equal or lesser processing time [26].

\section{B. Contributions}

The fundamental contribution of our work is a new analytical PLF method that, unlike the point estimate or linearization techniques, approximates the output distributions instead of the power equations. Linearization is built on an approximate input-to-output mapping that conserves statistical information. Input moments up to order $m$ are sufficient to explicitly compute the output moments up to order $m$. Our method achieves the same result for $m=2$ by approximating the output distributions with distributions that are fully characterized by their lower moments. The results are exact if the approximating distributions are exact.

We developed the method's implementation within the b/f framework. While it is also possible to develop an implementation in a centralized framework, we believe the recursive character of our decentralized approach is conceptually easy, modular, and efficient under normal operating conditions. It is im- 
portant to note the limitations of the deterministic b/f algorithm also apply to this extension.

Another contribution of our work is a comprehensive and conclusive comparison procedure of different methods that is based on the Wasserstein metric. To the best of our knowledge, the Wasserstein metric has yet to be used for power flow error evaluation. In literature, probabilistic estimation errors are usually compared separately for each output moment [7], [16], which can yield inconclusive results. The Wasserstein metric eliminates ambiguity by comparing the estimation errors directly in the sample space yielding a single error measurement for each output.

Empirically, the following potential contributions were found in estimating a well-documented radial IEEE 32-bus test system [22]. In comparison to linearization, uniformly better results were achieved at significant uncertainty levels. In comparison to PEMs, 1) uniformly better estimation of branch losses was achieved, 2) lower computational complexity by a single polynomial order was observed, and 3) marginally better estimates of nodal voltages was attained when the inputs were highly uncertain and the network was operating closer to its power limits. Based on this example, we can conclude that our method is potentially valuable for applications that require accurate estimates (especially power estimates) in the presence of uncertainty and applications that need to execute quickly (e.g., software used to explore the parameter space at many time instances or higher level Monte Carlo simulators).

\section{Organization}

The remainder of the paper is organized as follows: Section II defines the method domain by presenting the network model. The extended b/f method is presented in Section III. Section IV illustrates the method on a radial IEEE 32-bus test system. The paper concludes with Section V.

\section{Electrical Distribution Network Model}

In general, we make the following network assumptions: Assumption (Network Conditions):

A1. the network topology and branch impedances are fixed and known, the network is operating under normal conditions with no failures and filled power demands, and

A2. the voltages and currents are described by stationary stochastic processes.

By the first assumption, we can treat the network as a single phase system, where the nodes correspond to the buses and branches correspond to the lines, transformers, reactors, etc. By Assumption 2, we can model the network voltages and currents using phasor notation as complex random variables. Under these assumptions, we now define the detailed mathematical model.

\section{A. Notation}

The following notation is used throughout. Capital letters are used to denote random variables and matrices. The letter $i$ is reserved for the imaginary unit. Any vector $\left(x_{1}, \ldots, x_{n}\right)$ can be simply written as $\left(x_{k}\right)_{k \in\{1, \ldots, n\}}$ or as $x$. Moreover, if $x$ is complex, $\bar{x}:=(\operatorname{Re}[x], \operatorname{Im}[x])$ and its complex conjugate transpose is written as $x^{H}$. For random variables $X$ and $Y$, the expectation value is written as $\langle X\rangle$, the covariance is written as $\langle\langle X, Y\rangle\rangle$ (second argument is omitted whenever $X \equiv Y$ ), and the correlation is written as $\operatorname{corr}(X, Y)$. Moreover, if values of $Y$ are complex, additional subscripts $H$ and $T$ are used to specify how the covariance is evaluated:

$$
\begin{aligned}
&\langle\langle X, Y\rangle\rangle_{H}:=\left\langle(X-\langle X\rangle)(Y-\langle Y\rangle)^{H}\right\rangle \\
&\langle\langle X, Y\rangle\rangle_{T}:=\left\langle(X-\langle X\rangle)(Y-\langle Y\rangle)^{T}\right\rangle .
\end{aligned}
$$

Finally, $\nabla_{x} f(x, y)$ denotes the partial derivative of the function $f$ with respect to the vector $x$.

\section{B. Network Model}

Distribution networks often have a highly radial structure. Load flow analysis is commonly done by first decomposing the meshed networks into radial subnetworks and then applying radial analysis methods. Decomposition does not fall within the scope of this paper, hence the following assumption.

Assumption (Network Structure): The electrical distribution network is purely radial.

A radial network topology is equivalent to a directed tree graph. The root node corresponds to the power source and the other nodes correspond to the customers. The branches represent the power lines and are directed downstream towards the power source. The following is a formal description.

Consider a directed graph where the set of nodes is given by the finite set $\mathcal{N}=\{0,1, \ldots, N\}$ and the set of branches is defined by the set $\mathcal{B} \subset \mathcal{N} \times \mathcal{N}$, where each $(k, \ell) \in \mathcal{B}$ points from node $k$ to node $\ell$. For each node $k \in \mathcal{N}$, define the set of upstream nodes by the set $\mathcal{U}_{k}=\{j \in \mathcal{N} \mid \exists(j, k) \in \mathcal{B}\}$. Finally, impose a tree structure on the graph with node 0 at the root. This means the set $\mathcal{B}$ is such that there exists a single path (defined as a directed connection of edges) leading to node 0 from every other node. Any node in the graph that has an empty set of upstream nodes is called a leaf node. If there exists a path from node $k$ and to node $\ell$, than $k$ is said to be upstream of $\ell$ (denoted by $k>\ell$ ) and $\ell$ is said to be downstream of $k$ (denoted by $\ell<k$ ).

The graph nodes and branches are associated with the estimated network variables. For each node $k \in \mathcal{N}$, denote the estimated voltage by $V_{k} \in \mathbb{C}$, loop current by $J_{k} \in \mathbb{C}$, and the load by $S_{k}=P_{k}+i Q_{k} \in \mathbb{C}$. For any branch $b=(k, \ell) \in \mathcal{B}$, denote the estimated current by $I_{k} \in \mathbb{C}$ and power loss by $\Delta S_{k} \in \mathbb{R}$. The actual base voltage is given by $v_{0} \in \mathbb{R}$ and the actual branch impedance is given by $z_{k} \in \mathbb{C}$.

\section{Load Model}

The large size of a typical low voltage network prevents full statistical modeling of customer loads. In practice, these loads are estimated from the mean and variance of the customers' yearly energy consumptions.

Assumption (Customer Data): For each node $k \in \mathcal{N}$, denote the customers' actual load by $S_{0 k}=P_{0 k}+i Q_{0 k}$. The first two moments of the active and reactive powers (i.e., the quantities $\left\langle S_{0 k}\right\rangle,\left\langle\left\langle P_{0 k}\right\rangle\right\rangle$, and $\left.\left\langle\left\langle Q_{0 k}\right\rangle\right\rangle\right)$ are known.

Note, we make no assumptions regarding the correlations between different customers and between the active and reactive powers of the same customer. See the next section for our correlation model. 


\section{Correlation Model}

Load fluctuations are often correlated [9]. The correlations are important as they can significantly change the extremal voltages and currents. Modeling these correlations, however, is both computationally and practically difficult. Computationally, for approximate methods that rely on truncated Taylor series, the number of cross-terms increases combinatorially with the truncation order. Linearization methods circumvent this issue by ignoring higher order terms. PEM methods consider only pairwise cross-moments between the inputs [16]. The method presented herein is subjected to the same curse of dimensionality.

Practically, it is difficult to obtain quality synchronous data from all customers. Even if the necessary hardware was in place, the time varying topology and characteristics of distribution networks complicate the collection of statistical information. A categorical study (parametrizing customer dependencies) can be performed, but the challenge then becomes correctly preselecting the categories based on geographical location, customer type, tariff rate, etc.

Practitioners circumvent the challenges of correlation modeling by using dimensionless parameters, e.g., the diversity factor [27] used in practice to provide a factor of safety. Our correlation model (first introduced in [28]) is built around a contemporaneity factor $\gamma$ defined as the ratio of maximum current demand on the power station to the maximum individual current demand, i.e., $\gamma=\max \left|I_{0}\right| /\left(\sum_{k \in \mathcal{N}} \max \left|J_{k}\right|\right)$. Like the diversity factor, $\gamma$ is directly related to network robustness. Higher value of $\gamma$ means the power station must produce a higher current to meet the power demands.

With the purpose of creating an 1) easily tunable correlation model that 2) generates a wide range of $\gamma$ values for 3) various current phase angles, we assume the following current correlation structure.

Assumption (Current Correlation): For all $k, \ell \in \mathcal{N}, k \neq \ell$

$$
\begin{aligned}
\rho_{R} & =\operatorname{corr}\left(\operatorname{Re}\left[J_{k}\right], \operatorname{Re}\left[J_{\ell}\right]\right), \quad \rho_{I}=\operatorname{corr}\left(\operatorname{Im}\left[J_{k}\right], \operatorname{Im}\left[J_{\ell}\right]\right) \\
\rho_{R I} & =\operatorname{corr}\left(\operatorname{Re}\left[J_{k}\right], \operatorname{Im}\left[J_{\ell}\right]\right), \quad \rho=\operatorname{corr}\left(\operatorname{Re}\left[J_{k}\right], \operatorname{Im}\left[J_{k}\right]\right) .
\end{aligned}
$$

For the covariance matrix of $\bar{J}$ to be positive semidefinite, the values $\rho_{R}, \rho_{I}, \rho_{R I}$, and $\rho$ must satisfy

$$
0 \leq\left(\begin{array}{cc}
\left(1-\rho_{R}\right) \mathbf{I}_{N}+\rho_{R} \mathbf{1}_{N} & \left(\rho-\rho_{R I}\right) \mathbf{I}_{N}+\rho_{R I} \mathbf{1}_{N} \\
\left(\rho-\rho_{R I}\right) \mathbf{I}_{N}+\rho_{R I} \mathbf{1}_{N} & \left(1-\rho_{I}\right) \mathbf{I}_{N}+\rho_{I} \mathbf{1}_{N}
\end{array}\right)
$$

where $\mathbf{I}_{N}$ is the identity matrix of size $N$ and $\mathbf{1}_{N}$ is the $N \times N$ matrix of ones.

\section{BACKWARD/FORWARD ALGORITHM}

The probabilistic b/f algorithm proceeds similarly to its deterministic counterpart (see Fig. 1 for a basic flowchart). From the leaf nodes to the root, the backward sweep computes the first two moments of the branch currents and losses. From the root node to the leaf nodes, the forward sweep computes the first two moments of the nodal voltages and loads.

There is one main difference. In the deterministic version, the nodes update the loop currents by solving local power algebraic

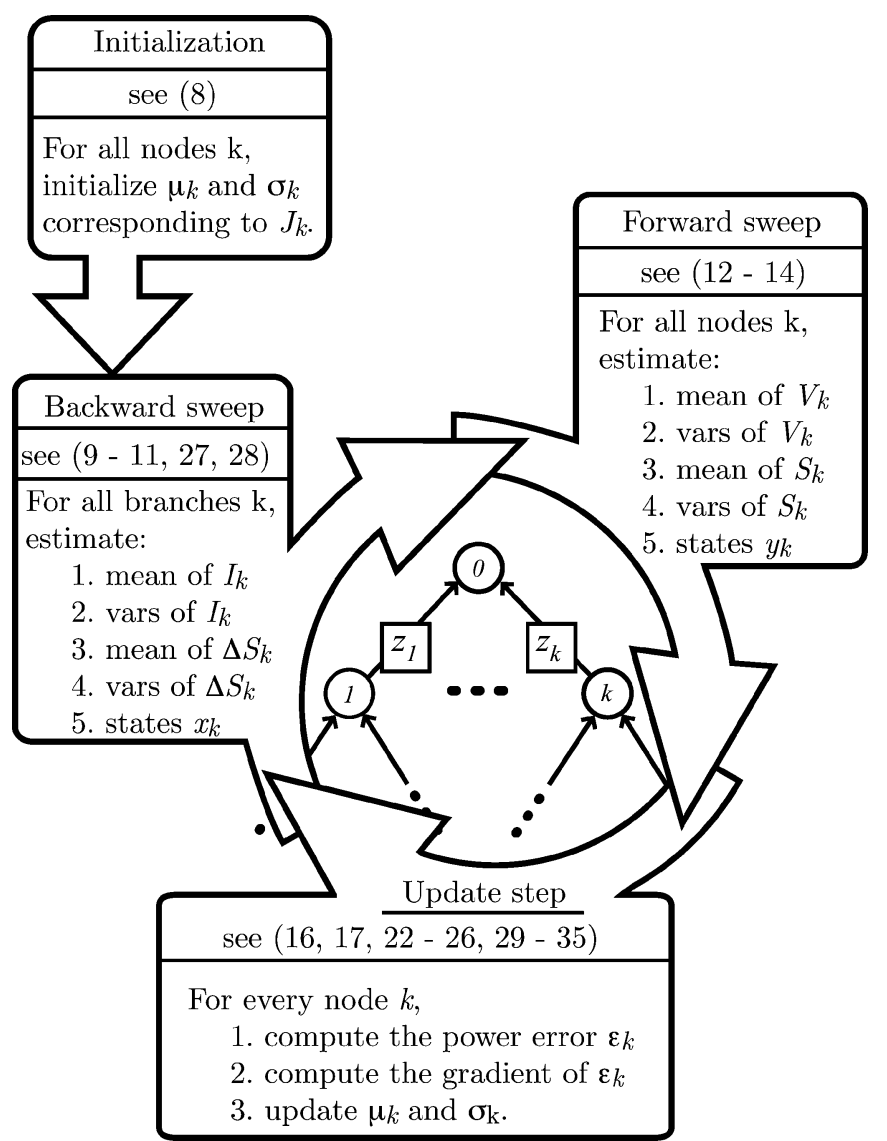

Fig. 1. Extended b/f method flowchart. In principle, this flowchart is identical to the flowchart of the deterministic b/f method. The loop currents are initialized at the beginning of the backward sweep and iteratively updated until the resulting power error is sufficiently small.

equations. In the probabilistic version, exact solution is not possible. So an approximation must be used. We assume this approximation is a function of the error gradient, which is computed in the forward sweep as well.

The majority of the algorithm is developed for a general class of distributions. In parts, however, the normal distribution is chosen as a representative member of this class.

Definition (GABF Method): The extended b/f method where the loop current vector $J$ is normally distributed is called the Gaussian backward/forward method (GABF).

The remaining details of the extended $b / f$ algorithm under the general and Gaussian assumptions are presented below.

\section{A. Initialization}

Many deterministic b/f algorithms begin by fixing the node voltages. The probabilistic algorithm begins by fixing the loop current distribution.

Assume that the loop current at node $k \in \mathcal{N}$ has the form

$$
J_{k}=\mu_{k}+\sigma_{k}^{T} W_{k}
$$

where $\mu_{k} \in \mathbb{C}, \sigma_{k} \in \mathbb{R}^{2}$ and $\sigma_{k} \geq 0$. The complex random variables $W_{k}$ have a zero mean and covariances defined by

$$
R_{1 s}:=\left\langle\left\langle W_{k}, W_{k}\right\rangle\right\rangle_{s}, \quad R_{2 s}:=\left\langle\left\langle W_{k}, W_{\ell}\right\rangle\right\rangle_{s}, \ell \neq k
$$


where $s \in\{H, T\}$. It can be shown that

$$
\begin{aligned}
& R_{1 H}=\left(\begin{array}{cc}
1 & -i \rho \\
i \rho & 1
\end{array}\right), \quad R_{1 T}=\left(\begin{array}{cc}
1 & i \rho \\
i \rho & -1
\end{array}\right) \\
& R_{2 H}=\left(\begin{array}{cc}
\rho_{R} & -i \rho_{R I} \\
i \rho_{R I} & \rho_{I}
\end{array}\right), R_{2 T}=\left(\begin{array}{cc}
\rho_{R} & i \rho_{R I} \\
i \rho_{R I} & -\rho_{I}
\end{array}\right)
\end{aligned}
$$

and

$$
\left\langle\left\langle J_{k}, J_{\ell}\right\rangle\right\rangle_{s}=\left\{\begin{array}{ll}
\sigma_{k}^{T} R_{2 s} \sigma_{\ell}, & \text { if } k \neq \ell \\
\sigma_{k}^{T} R_{1 s} \sigma_{\ell}, & \text { if } k=\ell
\end{array}, s \in\{H, T\} .\right.
$$

These formulas are used throughout to simplify the expressions in the $\mathrm{b} / \mathrm{f}$ sweeps.

The currents are initialized using the given customer power moments by the formulas

$$
\begin{aligned}
\mu_{k} & =\left\langle S_{0 k}^{H}\right\rangle / v_{0} \\
\sigma_{k} & =\left(\sqrt{\left\langle\left\langle P_{0 k}\right\rangle\right\rangle}, \sqrt{\left\langle\left\langle Q_{0 k}\right\rangle\right\rangle}\right) / v_{0} .
\end{aligned}
$$

Other formulas may produce better convergence properties (as is shown for the deterministic algorithm in [21]) but that is outside the scope of this paper.

\section{B. Backward Sweep}

The backward sweep begins at the leaf nodes and iteratively proceeds downstream. After obtaining the values of all upstream states, each node $k \in \mathcal{N}$ computes the value of its own state using the following simple formula:

$$
x(k)=\sum_{\ell \in \mathcal{U}_{k}} x(\ell)+\left(\begin{array}{c}
\mu_{k} \\
\sigma_{k} \\
\sigma_{k}^{T}\left(R_{1 s}-R_{2 s}\right) \sigma_{k}
\end{array}\right)_{s \in\{H, T\}} .
$$

The iterations terminate at the root node.

The means and covariances of the branch currents can be readily computed from the values of $x$.

Lemma 1: For any node $k \in \mathcal{N}$

$$
\begin{aligned}
\left\langle I_{k}\right\rangle & =x_{1}(k), \text { and, for } \ell \leq k \\
\left\langle\left\langle I_{k}, I_{\ell}\right\rangle\right\rangle_{s} & =x_{2}(k)^{T} R_{2 s} x_{2}(\ell)+x_{3 s}(k), s \in\{H, T\} .
\end{aligned}
$$

Note, we save the computation of the branch power losses for Section III-D.

\section{Forward Sweep}

The forward sweep begins at the root node, where the forward state $y(0)$ is initialized so that

$$
\begin{aligned}
y_{1}(0) & =v_{0} \\
\left(y_{2}(0), y_{3 s}(0), y_{4}(0), y_{5 s}(0)\right)_{s \in\{H, T\}} & =0 .
\end{aligned}
$$

The upstream nodes then evaluate their forward states using the following expression. For any $k \in \mathcal{N}$ and $\ell \in \mathcal{U}_{k}$

$$
y(\ell)=y(k)+\left(\begin{array}{c}
-z_{\ell} x_{1}(\ell) \\
z_{\ell} \\
z_{\ell} z_{\ell}^{s}+z_{\ell}^{s} y_{2}(k)+z_{\ell} y_{2}(k)^{s} \\
z_{\ell} x_{2}(\ell) \\
x_{3 s}(\ell) y_{3 s}(\ell)
\end{array}\right)_{s \in\{H, T\}}
$$

The iterations terminate at the leaf nodes.

The means and covariances of the node voltages and currents can be readily computed from the values of $y$.

Lemma 2: For any node $k \in \mathcal{N},\left\langle V_{k}\right\rangle=y_{1}(k)$

$$
\begin{aligned}
\left\langle\left\langle V_{k}, V_{k}\right\rangle\right\rangle_{s}= & y_{4}(k)^{s} R_{2 s} y_{4}(k)+y_{5 s}(k) \\
\left\langle\left\langle V_{k}, J_{k}\right\rangle\right\rangle_{s}= & -y_{4}(k)^{s} R_{2 s} \sigma_{k} \\
& -y_{2}^{s}(k) \sigma_{k}^{T}\left(R_{1 s}-R_{2 s}\right) \sigma_{k}
\end{aligned}
$$

where $s \in\{H, T\}$.

\section{Error Evaluation}

For all $k \in \mathcal{N}$, the error is taken to be the difference between the known load moments and the estimated moments produced by the currents, i.e.,

$$
\epsilon_{k}=\left(\begin{array}{c}
\left\langle\bar{S}_{k}\right\rangle \\
\left\langle\left\langle P_{k}\right\rangle\right\rangle \\
\left\langle\left\langle Q_{k}\right\rangle\right\rangle
\end{array}\right)-\left(\begin{array}{c}
\left\langle\bar{S}_{0 k}\right\rangle \\
\left\langle\left\langle P_{0 k}\right\rangle\right\rangle \\
\left\langle\left\langle Q_{0 k}\right\rangle\right\rangle
\end{array}\right) .
$$

The expectation value

$$
\left\langle S_{k}\right\rangle=\left\langle\left\langle V_{k}, J_{k}\right\rangle\right\rangle_{H}+\mu_{k}^{H} y_{1}(k) .
$$

In general, to compute the other components of the errors, third and fourth moments of the loop currents are also needed.

However, an attempt to compute the higher moments from higher moments of $J$ will simply produce another underdetermined system. So there are two options: 1) follow the linearization or PEM approach by approximating the power equations with simpler functions, or 2) approximate the distribution of the vector $(J, V)$ with a joint distribution that is characterized by its first two moments (e.g., the uniform or normal distributions, or any distribution they generate). This is the approach we take.

The first and second moments of $(J, V)$ are first derived. The following lemma will be useful for this purpose.

Lemma 3: Consider a complex random vector $X$. Then

$$
\begin{aligned}
& 2\langle\langle\operatorname{Re}[X], \operatorname{Re}[X]\rangle\rangle=\operatorname{Re}\left[\langle\langle X\rangle\rangle_{H}+\langle\langle X\rangle\rangle_{T}\right] \\
& 2\langle\langle\operatorname{Re}[X], \operatorname{Im}[X]\rangle\rangle=\operatorname{Im}\left[\langle\langle X\rangle\rangle_{T}-\langle\langle X\rangle\rangle_{H}\right] \\
& 2\langle\langle\operatorname{Im}[X], \operatorname{Im}[X]\rangle\rangle=\operatorname{Re}\left[\langle\langle X\rangle\rangle_{H}-\langle\langle X\rangle\rangle_{T}\right] .
\end{aligned}
$$

For all $k \in \mathcal{N}$, begin by separating the real and imaginary parts of the voltage $V_{k}$ and the current $J_{k}$ to form the vector

$$
U_{k}=\overline{\left(J_{k}, V_{k}\right)} .
$$


The mean of $U_{k}$ has the form

$$
\left\langle U_{k}\right\rangle=\overline{\left(\mu_{k}, y_{1}(k)\right)}
$$

and, by Lemma 3 , the covariance of $U_{k}$, denoted by $\Sigma_{k}$, has the form

$$
\Sigma_{k}=\frac{1}{2}\left(\begin{array}{cc}
\operatorname{Re}\left[\Gamma_{k H}+\Gamma_{k T}\right] & \operatorname{Im}\left[\Gamma_{k T}-\Gamma_{k H}\right] \\
\operatorname{Im}\left[\Gamma_{k T}-\Gamma_{k H}\right]^{T} & \operatorname{Re}\left[\Gamma_{k H}-\Gamma_{k T}\right]
\end{array}\right)
$$

where $\Gamma_{k s}:=\left\langle\left\langle\left(J_{k}, V_{k}\right)\right\rangle\right\rangle_{s}, s \in\{H, T\}$. Note $\Gamma_{k s}$ has already been computed in the backward and forward sweeps in Sections III-B and III-C.

Next assume the joint distribution of $(J, V)$ is normal. ${ }^{1}$ Then, according to [29, Theorem 3.3.1], the variances of $P_{k}$ and $Q_{k}$ are given by

$$
\begin{aligned}
\left\langle\left\langle P_{k}\right\rangle\right\rangle= & 2 \operatorname{trace}\left[A_{P} \Sigma_{k} A_{P} \Sigma_{k}\right] \\
& +4\left\langle U_{k}\right\rangle^{T} A_{P} \Sigma_{k} A_{P}\left\langle U_{k}\right\rangle \\
\left\langle\left\langle Q_{k}\right\rangle\right\rangle= & 2 \operatorname{trace}\left[A_{Q} \Sigma_{k} A_{Q} \Sigma_{k}\right] \\
& +4\left\langle U_{k}\right\rangle^{T} A_{Q} \Sigma_{k} A_{Q}\left\langle U_{k}\right\rangle
\end{aligned}
$$

where

$$
2 A_{P}=\left(\begin{array}{cccc}
0 & 1 & 0 & 0 \\
1 & 0 & 0 & 0 \\
0 & 0 & 0 & 1 \\
0 & 0 & 1 & 0
\end{array}\right), 2 A_{Q}=\left(\begin{array}{cccc}
0 & 0 & 0 & 1 \\
0 & 0 & -1 & 0 \\
0 & -1 & 0 & 0 \\
1 & 0 & 0 & 0
\end{array}\right) .
$$

This concludes the derivation of formulas required to compute the error.

Remark 1: The branch power losses $\Delta S_{k}=\operatorname{Re}\left(z_{k}\right) I_{k}^{H} I_{k}$ can be computed in the same way:

$$
\begin{aligned}
\left\langle\Delta S_{k}\right\rangle & =\operatorname{Re}\left(z_{k}\right)\left\langle\left\langle I_{k}\right\rangle\right\rangle_{H} \\
\left\langle\left\langle\Delta S_{k}\right\rangle\right\rangle & =2 \operatorname{trace}\left[\left\langle\left\langle\bar{I}_{k}\right\rangle\right\rangle^{2}\right]+4 \bar{y}_{1}(k)^{T}\left\langle\left\langle\bar{I}_{k}\right\rangle\right) \bar{y}_{1}(k) .
\end{aligned}
$$

\section{E. Update Step}

Following the b/f sweeps, the loop currents at every node are replaced by new estimates to reduce the error. There exists no expression for the moments in terms of the load error (as does in the deterministic case), so a linear approximation is used. In this section, we present the gradient of $\epsilon$ as a function of the local $\mathrm{b} / \mathrm{f}$ states, and implement it in a decentralized Newton-Rhapson step to update the customer currents.

For all $k \in \mathcal{N}$, the gradient of $\left(J_{k}, V_{k}\right)$ is sufficient to compute the gradient of $\epsilon$. Independent of the distribution, this gradient is given by the following recursive formulas:

$$
\begin{aligned}
\frac{\partial\left\langle V_{k}\right\rangle}{\partial \mu_{k}}= & -y_{2}(k)(1 \quad i)^{T} \\
\frac{\partial\left\langle\left\langle V_{k}\right\rangle\right\rangle_{s}}{\partial \sigma_{k}}= & 2 \operatorname{Re}\left[\left(R_{1 s}-R_{2 s}\right)\right] \sigma_{k} y_{3 s}(k) \\
& +y_{2}(k) R_{2 s} y_{4}(k)+\left(y_{2}(k) R_{2 s} y_{4}(k)\right)^{s}
\end{aligned}
$$

${ }^{1}$ If $J_{k}$ is truly Gaussian, then $V_{k}$ is also Gaussian and GABF is exact. GABF will not be exact for distributions that are not closed under addition.

$$
\begin{aligned}
\frac{\partial\left\langle\left\langle V_{k}, J_{k}\right\rangle\right\rangle_{s}}{\partial \sigma_{k}}= & -y_{2}^{s}(k) R_{2 s} \sigma_{k}-\left(y_{4}^{s}(k) R_{2 s}\right)^{T} \\
& -y_{2}^{s}(k)\left(R_{1 s}+R_{1 s}^{T}-R_{2 s}-R_{2 s}^{T}\right) \sigma_{k}
\end{aligned}
$$

where $s \in\{H, T\}$. Note, all null components of the gradient and the derivatives of $\left\langle J_{k}\right\rangle$ and $\left\langle\left\langle J_{k}\right\rangle\right\rangle$ are omitted as they follow directly from (7).

The gradient of $\left\langle S_{k}\right\rangle$ follows directly from (17). If we again assume $(J, V)$ to be normal, then the gradient of the active and reactive load variances can be computed from (24) and (25) using the trace derivative formula [30]:

$$
\begin{aligned}
& \frac{\partial\left\langle\left\langle P_{k}\right\rangle\right\rangle}{\partial \mu_{k}}=8 \frac{\partial\left\langle U_{k}\right\rangle^{T}}{\partial \mu_{k}} A_{P} \Sigma_{k} A_{P}\left\langle U_{k}\right\rangle \\
& \frac{\partial\left\langle\left\langle P_{k}\right\rangle\right\rangle}{\partial \sigma_{k i}}=\operatorname{trace}\left[4 A_{P}\left(\Sigma_{k}+\left\langle U_{k}\right\rangle\left\langle U_{k}\right\rangle^{T}\right) A_{P} \frac{\partial \Sigma_{k}}{\partial \sigma_{k i}}\right] .
\end{aligned}
$$

Note, the expressions for $Q_{k}$ are identical with $A_{Q}$ substituted for $A_{P}$.

A decentralized Newton-Rhapson method can now be used to update the customer current estimates using the power error $\epsilon_{k}$ and its derivative $\nabla_{\mu_{k}, \sigma_{k}}\left(\epsilon_{k}\right)$. The mean and standard deviation of the customer currents are updated by

$$
\left(\mu_{k}, \sigma_{k}\right) \rightarrow\left(\mu_{k}, \sigma_{k}\right)-\nabla_{\mu_{k}, \sigma_{k}}^{-1}\left(\epsilon_{k}\right) \epsilon_{k} .
$$

\section{COMPARISON STUdY}

GABF was tested on the radial 12.66-kV IEEE 32-bus test system first published in [22]. Only the basic configuration in [22] was considered, i.e., the nonlinear loss reductions considered therein were ignored. This system was chosen to be small enough for simple Monte Carlo analysis and large enough to test accuracy and convergence. Load variances, which are not listed in [22], were defined parametrically as percentages of the load means, i.e., for $p \in\{5,10,20\}$ and for all $k \in \mathcal{N}$, $\left\langle\left\langle P_{0 k}\right\rangle\right\rangle=(p / 100)^{2}\left\langle P_{0 k}\right\rangle^{2}$ and $\left\langle\left\langle Q_{0 k}\right\rangle\right\rangle=(p / 100)^{2}\left\langle Q_{0 k}\right\rangle^{2}$. A Wasserstein metric was used to evaluate the results. The remaining details are given below.

\section{A. Method Evaluation Procedure}

GABF was compared to linearization and the PEM in [16], which allows for correlated loads. A thorough Monte Carlo simulation (300 000 runs) was used for reference. The comparison procedure is outlined in Fig. 2.

One difference between our method and linearization or PEM is the correlation model, which can significantly effect the output estimates. Linearization and PEM both impose correlations on customer loads, whereas our method imposes correlations on the corresponding loop currents. So the only fair comparison is when the input correlations of all methods are correct. To account for some loading synchrony, we consider the case with a small positive correlation in real loop currents and all other correlations zero, i.e., $\rho_{R}=0.3$ and $\rho=\rho_{I}=\rho_{R I}=0$. Using GABF and a Gaussian Monte Carlo simulation (300000 runs), we then compute the off-diagonal elements of the matrix $\left\langle\left\langle\overline{S_{0}}\right\rangle\right\rangle$ that result from Gaussian currents 


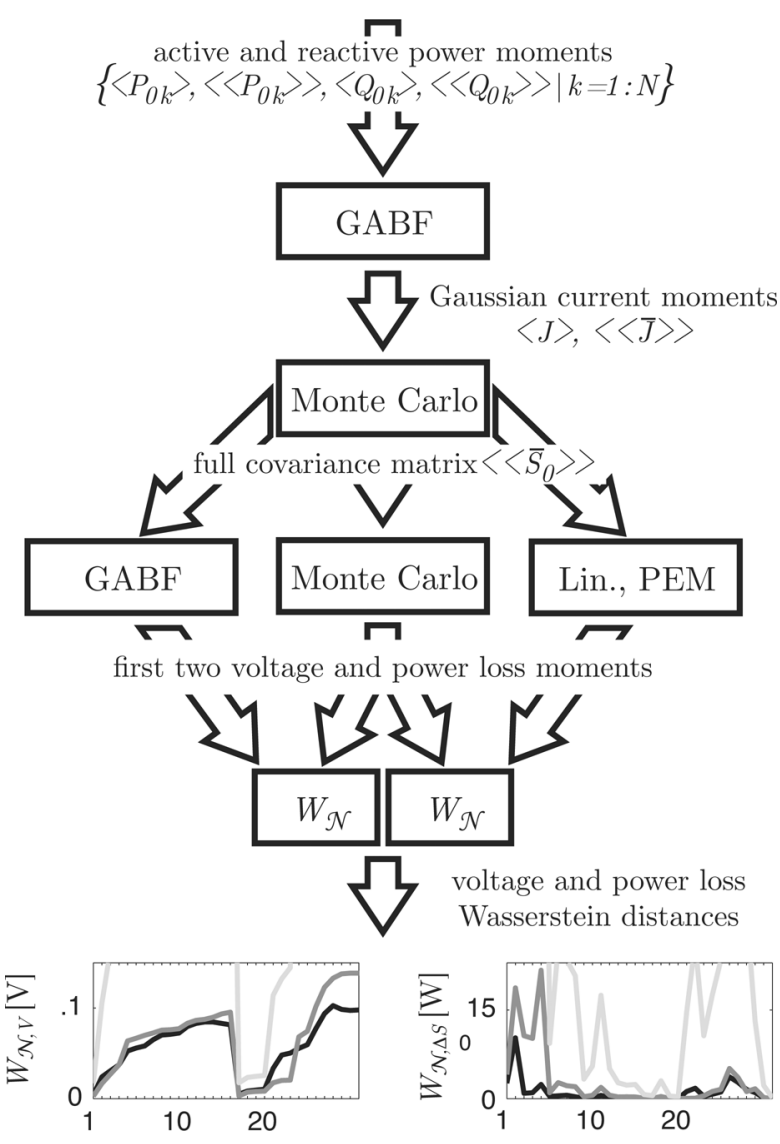

Fig. 2. Procedure used to compare GABF, PEM, and the linearization method. The procedure ensures the input correlations used by all methods are correct.

and their assumed correlations. This covariance matrix and the mean power vector $\left\langle\overline{S_{0}}\right\rangle$ are then input to the linearization method, the PEM, and the reference Monte Carlo simulator (300 000 runs), which draws power samples from a multivariate normal distribution (characterized by the computed mean and variance of $\overline{S_{0}}$ ). The estimates of the different methods are then compared to the reference. Note, the reference loop currents generated by the final Monte Carlo simulation are not Gaussian (implying GABF is in this case only an approximation).

Comparison of the network estimates is complicated by the number of associated variables. Each random variable of interest is described by its first two moments leading to either 2 or 5 quantities depending on whether the random variable is real or complex. Comparing these moments separately can be misleading. Instead we use the moments to define an underlying normal random variable and then use the multivariate Wasserstein metric [31] to measure the distance between the estimates and the reference values.

The Wasserstein metric is readily used in the literature to measure the distance between two separate stochastic processes [32]. If a random variable $X_{1}$ is an estimate of $X_{2}$, then the Wasserstein distance of $X_{1}$ from $X_{2}$ represents the smallest average prediction error one can hope for. This distance has a simple expression if the two random variables are Gaussian [31]:

$\mathcal{W}_{\mathcal{N}}=\sqrt{\left\|m_{1}-m_{2}\right\|_{2}^{2}+\operatorname{trace}\left[C_{1}+C_{2}-2\left(\sqrt{C_{1}} C_{2} \sqrt{C_{1}}\right)^{1 / 2}\right]}$ where, for $k=1,2, m_{k}$ and $C_{k}$ are the expected value and covariance matrix of $X_{k}$, respectively. In the sequel, we compute the Gaussian distances $\mathcal{W}_{\mathcal{N}, V}$ and $\mathcal{W}_{\mathcal{N}, \Delta S}$ to compare the voltage and power loss estimates of various methods to the reference Monte Carlo simulations.

\section{B. Results}

Intuition suggests that any gains of GABF over linearization or PEM will be greater when the nonlinear effects are greater. So we expect better relative performance at higher power uncertainties or lower operational voltages. We also expect GABF to better predict power losses, which are themselves nonlinear with respect to the branch currents.

Nodal voltages and branch power losses were estimated using all three methods. The Wasserstein distances between the estimates and the Monte Carlo references at every node are plotted in Fig. 3. Plots (a), (b) and (e), (f) correspond to voltage errors, and plots (c), (d) and (g), (h) correspond to power loss errors. The figure is organized by the operating regime. Four scenarios were considered:

S1. 5\% uncertainty, $v_{0}=12.66 \mathrm{kV}$ [Fig. 3(a), (c)];

S2. $20 \%$ uncertainty, $v_{0}=12.66 \mathrm{kV}$ [Fig. 3(b), (d)];

S3. $5 \%$ uncertainty, $v_{0}=8.00 \mathrm{kV}[$ Fig. $3(\mathrm{e}),(\mathrm{g})]$;

S4. $20 \%$ uncertainty, $v_{0}=8.00 \mathrm{kV}$ [Fig. 3(f), (h)].

The plots show how the three methods compare at every node and how the errors change as the distance from the root node increases. GABF appears to be the most accurate in general; however, accuracy of the PEM is often comparable for voltage estimates. Power loss errors grow inversely with the distance from the root node while the voltage errors grow proportionally (see [22] for the network topology).

The average Wasserstein distances for each scenario and method are listed in the center table of Fig. 3. The average results further support our expectations. Power losses are better approximated by GABF across all regimes (approx. 40\%-70\% improvement over PEM and 80\%-90\% improvement over linearization). In all scenarios, both GABF and PEM better estimate voltages than linearization (50\% to $90 \%$ improvement). In Scenario 4, GABF further outperforms PEM in voltage estimation by a $30 \%$ margin.

Next we compared the computational complexity for this example. The linearization method and the PEM implemented herein rely on a deterministic b/f algorithm. We compare the convergence of this deterministic b/f algorithm to the convergence of GABF. Since the two make comparable steps in each iteration, the difference in convergence rates gives an idea of the difference in complexities. It is expected that GABF will exhibit slower convergence as the update step is not exact (i.e., it is based on Newton-Rhapson approximation instead of an explicit solution).

Under normal operating conditions (all regimes considered in this section), the convergence rates of the deterministic $b / f$ method and GABF were the same (see Fig. 4), suggesting their computational complexities have the same polynomial order. Hence, the observed computational complexity of GABF is comparable to linearization and smaller by a polynomial degree than the PEM complexity, which requires approximately two 

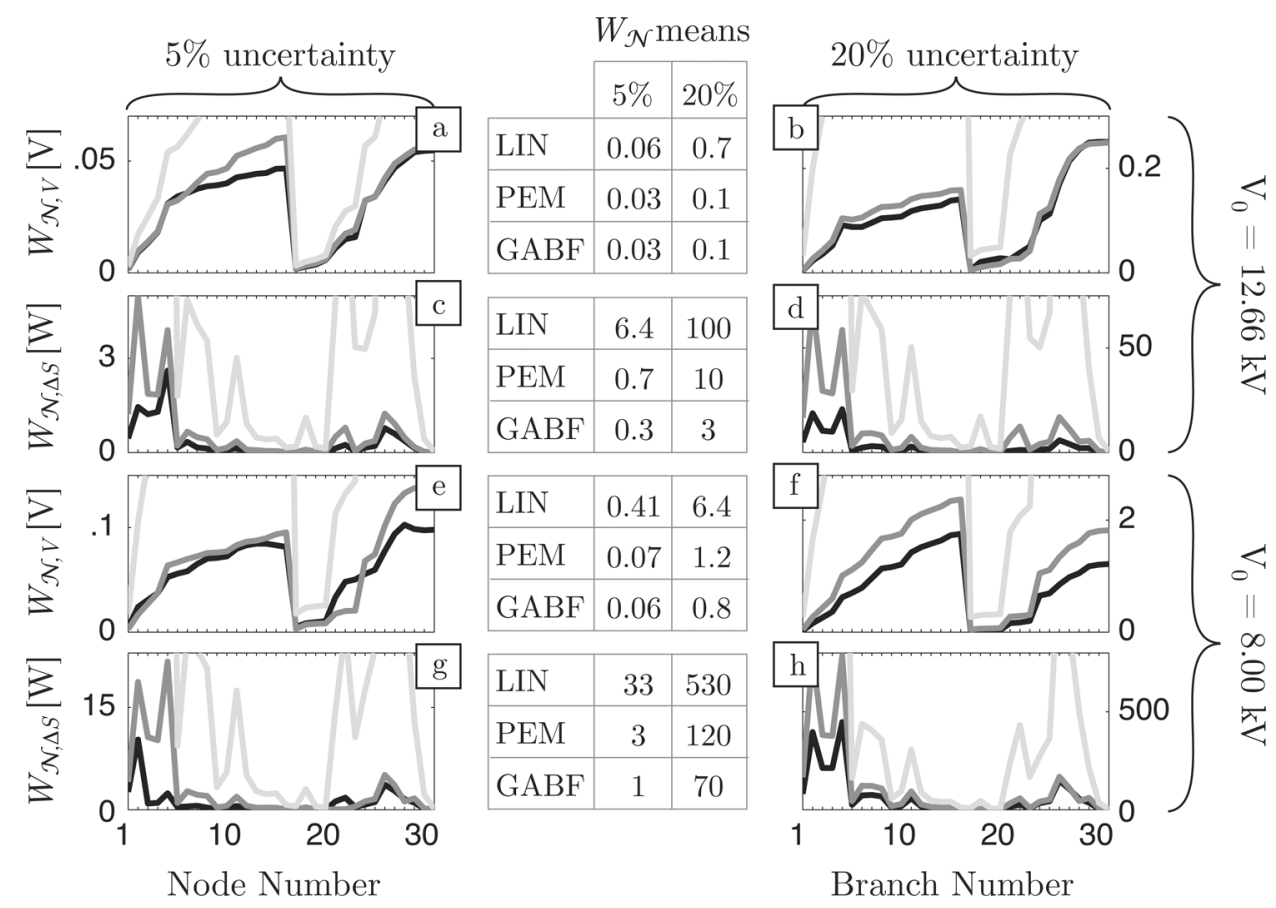

Fig. 3. Wasserstein errors for node voltage and power loss estimates produced by GABF (black), PEM (dark grey), and the linearization method (light grey). Plots on the left and right sides of the table correspond to power uncertainties of 5\% and 20\%, respectively. Plots (a)-(d) correspond to the high base voltage scenarios, and plots (e)-(h) correspond to the low base voltage scenarios. The center table indicates the mean errors for every scenario and method.

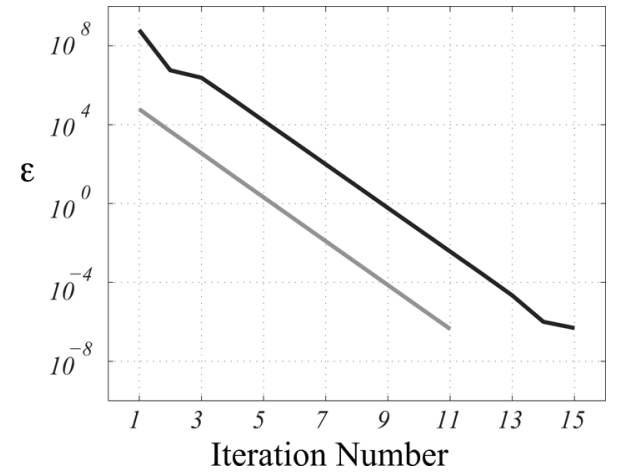

Fig. 4. Typical convergence plot showing power errors at iterations of GABF (black) and the deterministic $\mathrm{b} / \mathrm{f}$ method (grey) for $v_{0}=12.66 \mathrm{kV}$ and power uncertainty equal to $10 \%$ of the mean.

simulations for each node. In other scenarios, whenever the probability of a network power limit violation increased (either by increasing load uncertainty or by lowering the base voltage), GABF did diverge. Scalability of the b/f sweeps was further tested on much larger systems in [33].

\section{CONCLUSION}

A new analytical PLF method was introduced. Its novelty is in approximating output distributions with characterizable distributions instead of approximating the power flow equations. The method was built on an efficient $b / f$ algorithm for radial networks.
A comprehensive case study of a radial IEEE 32-bus test system showed that the method does well at retaining the nonlinear effects of the input-output network mapping. In a comparison with a PEM and a linearization method, uniformly more accurate estimates of power losses were achieved. Nodal voltages were also better estimated in scenarios with high input uncertainties and lower base voltage. Improvements in the computational complexity over PEM were also observed.

Extension of the method to meshed networks is part of the future work. This will entail developing a break-point-based decomposition similar to that of [17]. Alternatively, the implementation could be developed in a centralized framework that is free of radial constraints, e.g., the current injection method in [26].

There is also the potential to further increase the method's accuracy through integration with existing simulation methods. For instance, other output probabilities could be approximated with a mixture of Gaussian distributions.

\section{APPENDIX}

In this section, we prove the three new lemmas introduced in the paper. Note, the remainder of the results follow directly from these lemmas.

Throughout, summations over network paths are used. Recall the notation from Section II-B, $k<n$ (equivalently, $n>k$ ) if there is a path from $n$ to $k$. The sum $\sum_{k>n}$ is taken over the set $\{k \in \mathcal{N} \mid k>n \vee k=n\}$ and the sum $\sum_{k>n, k \ngtr \ell}$ is taken over the set $\{k \in \mathcal{N} \mid k>n \vee k=n\} \backslash\{k \in \mathcal{N} \mid k>\ell\}$. The other permutations of this notation used below should be clear.

\section{A. Proof of Lemma 1}

The first part of the lemma (the formula for the current means) follows directly from Kirchoff's current laws. So its proof is 
here omitted. We now focus on the covariance formula. For any $k \in \mathcal{N}$, write out the variance of $I_{k}$ explicitly in terms of the injected currents and apply (7) to obtain

$$
\begin{aligned}
\left\langle\left\langle I_{k}\right\rangle\right\rangle_{s} & =\sum_{n \geq k}\left(\sigma_{n}^{T}\left(R_{1 s}-R_{2 s}\right) \sigma_{n}+\sum_{j \geq k} \sigma_{n}^{T} R_{2 s} \sigma_{j}\right) \\
& =x_{3 s}(k)+x_{2}(k)^{T} R_{2 s} x_{2}(k)
\end{aligned}
$$

where the last equality follows by inspection from the backward state equations.

Next, for any $\ell<k$, the covariance of $I_{k}$ with $I_{\ell}$ is derived as follows. First, separate the covariance as

$$
\left\langle\left\langle I_{k}, I_{\ell}\right\rangle\right\rangle_{s}=\left\langle\left\langle I_{k}, I_{k}-I_{\ell}\right\rangle\right\rangle_{s}+\left\langle\left\langle I_{k}\right\rangle\right\rangle_{s} .
$$

The first term on the right-hand side is a covariance of different loop currents. Therefore, by (7)

$$
\left\langle\left\langle I_{k}, I_{\ell}\right\rangle\right\rangle_{s}=\sum_{n \geq k} \sum_{\substack{j \geq \ell \\ j \leq k-1}} \sigma_{n}^{T} R_{2 s} \sigma_{j}+\left\langle\left\langle I_{k}\right\rangle\right\rangle_{s} .
$$

Combining this expression with (37) and utilizing the backward state formulas yields the desired result:

$$
\begin{aligned}
\left\langle\left\langle I_{k}, I_{\ell}\right\rangle\right\rangle_{s}= & \sum_{n \geq k} \sigma_{n}^{T}\left(R_{1 s}-R_{2 s}\right) \sigma_{n} \\
& +\left(\sum_{n \geq k} \sigma_{n}\right)^{T} R_{2 s}\left(\sum_{n \geq \ell} \sigma_{n}\right) \\
= & x_{3 s}(k)+x_{2}(k)^{T} R_{2 s} x_{2}(\ell) .
\end{aligned}
$$

\section{B. Proof of Lemma 2}

Here we prove the expressions for $s=T$. When $s=H$, the expressions are derived in the same way.

The voltage mean formula follows from direct application of Kirchoff's voltage laws, so its proof is here omitted. We begin with the voltage covariances. Rewrite the voltages in terms of the branch currents and apply Lemma 1 to obtain the equations

$$
\begin{aligned}
\left\langle\left\langle V_{k}, V_{k}\right\rangle\right\rangle_{T}= & \sum_{n \leq k} z_{n}^{2}\left(x_{3 T}(n)+x_{2}(n)^{T} R_{2 T} x_{2}(n)\right) \\
& +2 \sum_{j<n} z_{n} z_{j}\left(x_{3 T}(n)+x_{2}(n)^{T} R_{2 T} x_{2}(j)\right) \\
= & \sum_{n \leq k} x_{3 T}(n)\left(z(n)^{2}+2 z(n) \sum_{j<n} z(j)\right) \\
& +\sum_{n \leq k} \sum_{j \leq k} z(n) z(j) x_{2}^{T}(n) R_{2 T} x_{2}(j) .
\end{aligned}
$$

The final form of the voltage covariance follows from direct substitution of the forward states into the above equation.
Next we prove the formula for the covariance of $V_{k}$ with $J_{k}$. Rewrite the voltages in terms of the loop currents and apply (7) to obtain the equations

$$
\begin{aligned}
\left\langle\left\langle V_{k}, J_{k}\right\rangle\right\rangle_{T}= & -\sum_{n \leq k} \sum_{j \geq n} z(n) \sigma_{n}^{T} R_{2 T} \sigma_{k} \\
& -\sum_{n \leq k} z(n) \sigma_{k}^{T}\left(R_{1 T}-R_{2 T}\right) \sigma_{k} \\
= & -\left(\sum_{n \leq k} z(n)\left(\sum_{j \geq n} \sigma_{n}^{T}\right)\right) R_{2 T} \sigma_{k} \\
& -\sigma_{k}^{T}\left(R_{1 T}-R_{2 T}\right) \sigma_{k}\left(\sum_{n \leq k} z(n)\right) .
\end{aligned}
$$

The final formula follows from direct substitution of forward state definitions.

\section{Proof of Lemma 3}

We prove only the first expression, as the others follow in the same way. Consider two complex random variables $X=$ $X_{R}+i X_{I}$ and $Y=Y_{R}+i Y_{I}$ :

$$
\begin{aligned}
\operatorname{Re}\left[\langle\langle X, Y\rangle\rangle_{H}\right] & =\left\langle\left\langle X_{R}, Y_{R}\right\rangle\right\rangle+\left\langle\left\langle X_{I}, Y_{I}\right\rangle\right\rangle \\
\operatorname{Re}\left[\langle\langle X, Y\rangle\rangle_{T}\right] & =\left\langle\left\langle X_{R}, Y_{R}\right\rangle\right\rangle-\left\langle\left\langle X_{I}, Y_{I}\right\rangle\right\rangle .
\end{aligned}
$$

It follows that

$$
2\left\langle\left\langle X_{R}, Y_{R}\right\rangle\right\rangle=\operatorname{Re}\left[\langle\langle X, Y\rangle\rangle_{H}+\langle\langle X, Y\rangle\rangle_{T}\right]
$$

\section{REFERENCES}

[1] Q. Zhou and J. Bialek, "Generation curtailment to manage voltage constraints in distribution networks," IET Gen., Transm. Distrib., vol. 1, pp. 492-498, 2007

[2] J. Wood, "Under pressure [renewable energy generation]," Power Eng., vol. 19, no. 2, pp. 12-15, 2005.

[3] P. Chen, Z. Chen, and B. Bak-Jensen, "Probabilistic load flow: A review," in Proc. 3rd Int. Conf. Electric Utility Deregulation and Restructuring and Power Technologies, 2008.

[4] H. Yu, C. Y. Chung, K. P. Wong, H. W. Lee, and J. H. Zhang, "Probabilistic load flow evaluation with hybrid Latin hypercube sampling and Cholesky decomposition," IEEE Trans. Power Syst., vol. 24, no. 2, pp. 661-667, May 2009.

[5] S. Chun-Lien, "Probabilistic load-flow computation using point estimate method," IEEE Trans. Power Syst., vol. 20, no. 4, pp. 1843-1851, Nov. 2005

[6] X. Li, Y. Li, and S. Zhang, "Analysis of probabilistic optimal power flow taking account of the variation of load power," IEEE Trans. Power Syst., vol. 23, no. 3, pp. 992-999, Aug. 2008.

[7] J. M. Morales and J. Perez-Ruiz, "Point estimate schemes to solve the probabilistic power flow," IEEE Trans. Power Syst.., vol. 22, no. 4, pp. 1594-1601, Nov. 2007.

[8] A. M. L. da Silva and V. L. Arienti, "Probabilistic load flow by a multilinear simulation algorithm," Proc. Inst. Elect. Eng. C: Gen., Transm., Distrib., vol. 137, no. 4, pp. 276-282, Jul. 1990.

[9] R. Cicoria, G. L. Fracassi, G. Gambelli, M. Mazzoni, and W. Palenzona, "Load flow calculations on distribution networks by using a statistical approach," in Proc. 14th Int. Conf. Exhib. Electricity Distribution. Part 1: Contributions, CIRED, 1997.

[10] B. Borkowska, "Probabilistic load flow," IEEE Trans. Power App. Syst., vol. PAS-93, pp. 752-759, 1974.

[11] R. N. Allan and M. R. G. Al-Shakarchi, "Probabilistic a.c. load flow," Proc. Inst. Elect. Eng., vol. 123, no. 6, pp. 531-536, 1976. 
[12] A. Dimitrovski and R. Ackovski, "Probabilistic load flow in radial distribution networks," in Proc. Transmission and Distribution Conf., 1996.

[13] R. N. Allan, A. M. L. da Silva, and R. C. Burchett, "Evaluation methods and accuracy in probabilistic load flow solutions," IEEE Trans. Power App. Syst., vol. PAS-100, no. 5, pp. 2539-2546, 1981.

[14] L. Xiaoming, C. Xiaohui, Y. Xianggen, X. Tieyuan, and L. Huagang, "The algorithm of probabilistic load flow retaining nonlinearity," in Proc. Int. Conf. Power System Technology, 2002.

[15] H. Hong, "An efficient point estimate method for probabilistic analysis," Reliab. Eng. Syst. Safety, vol. 59, pp. 261-267, 1998.

[16] J. Morales, L. Baringo, A. Conejo, and R. Minguez, "Probabilistic power flow with correlated wind sources," IET Gen., Transm., Distrib., vol. 4, no. 5, pp. 641-651, 2010.

[17] D. Shirmohammadi, H. Hong, A. Semlyen, and G. Luo, "A compensation-based power flow method for weakly meshed distribution and transmission networks," IEEE Trans. Power Syst., vol. 3, no. 2, pp. 753-762, May 1988.

[18] G. X. Luo and A. Semlyen, "Efficient load flow for large weakly meshed networks," IEEE Trans. Power Syst., vol. 5, no. 4, pp. 1309-1316, Nov. 1990.

[19] C. S. Cheng and D. Shirmohammadi, "A three-phase power flow method for real-time distribution system analysis," IEEE Trans. Power Syst., vol. 10, no. 2, pp. 671-679, May 1995.

[20] R. Ciric, A. Feltrin, and L. Ochoa, "Power flow in four-wire distribution networks-general approach," IEEE Trans. Power Syst., vol. 18, no. 4, pp. 1283-1290, Nov. 2003.

[21] A. Augugliaro, L. Dusonchet, S. Favuzza, M. G. Ippolito, and E. R. Sanseverino, "Some improvements in solving radial distributions networks through the backward/forward method," in Proc. IEEE Russia Power Tech., Jul. 2005.

[22] M. E. Baran and F. F. Wu, "Network reconfiguration in distribution systems for loss reduction and load balancing," IEEE Trans. Power Del., vol. 4, no. 2, pp. 1401-1407, Apr. 1989.

[23] J.-C. Wang, H.-D. Chiang, and G. Darling, “An efficient algorithm for real-time network reconfiguration in large scale unbalanced distribution systems," IEEE Trans. Power Syst., vol. 11, no. 1, pp. 511-517, Feb. 1996.

[24] L. Araujo, D. Penido, S. Carneiro, J. Pereira, and P. Garcia, "A comparative study on the performance of TCIM full Newton versus backward-forward power flow methods for large distribution systems," in Proc. Power Systems Conf. Expo., 2006.

[25] Y. Yubin, D. Wang, W. Zhiliang, and C. Yong, "Convergence analysis on the power flow methods for distribution networks with small impedance branches," in Proc. 3rd Int. Conf. Electric Utility Deregulation and Restructuring and Power Technologies, 2008.

[26] P. A. N. Garcia, J. Pereira, J. S. Carneiro, V. da Costa, and N. Martins, "Three-phase power flow calculations using the current injection method," IEEE Trans. Power Syst., vol. 15, no. 2, pp. 508-514, May 2000.

[27] IEEE Recommended Practice for Electric Power Distribution for Industrial Plants, Red Book, IEEE standard 141 (tm) ed., 1993.

[28] E. Janeček and P. Prautsch, "Closed form estimation of boundary values in electrical distribution networks," in Proc. 16th IFAC World Congr., 2006.
[29] F. Štulajter, Odhady v Náhodných Procesoch, alfa, 1989.

[30] D. S. Bernstein, Matrix Mathematics: Theory, Facts, and Formulas With Application to Linear Systems Theory. Princeton, NJ: Princeton Univ. Press, 2005.

[31] C. R. Givens and R. M. Shortt, "A class of Wasserstein metrics for probability distributions," Michigan Math. J., vol. 31, pp. 231-240, 1984.

[32] D. Thorsley and E. Klavins, "Approximating stochastic biochemical processes with Wasserstein pseudometrics," IET Syst. Biol., vol. 4, no. 3, pp. 193-211, Nov. 2009.

[33] J. Krynsky, E. Janeček, and P. Janeček, "A new stochastic model of the electric distribution network," in Proc. Int. Conf. Probabilistic Methods Applied to Power Systems, 2004, pp. 284-288.

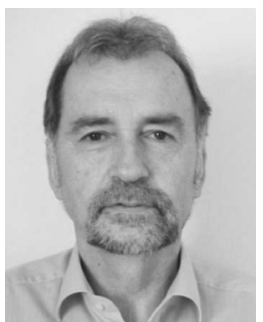

Eduard Janeček received the Ph.D. degree in electrical engineering from the Czech Technical University, Prague, Czech Republic, in 1987.

After graduation, he joined the faculty at the University of West Bohemia, Pilsen, Czech Republic, where he currently serves as an Associate Professor and director of the Information and Control Center and as the head of the Applied Cybernetics Center. His research has produced numerous methods and algorithms for modeling, simulation, diagnostics, and reliability analysis of energy systems that are in use by international industry partners such as AREVA NP GmbH and Škoda Power a Doosan Company, and regional industry partners such as the electrical distribution company CEZ group and the Czech transmission security operator ČEPS a.s.

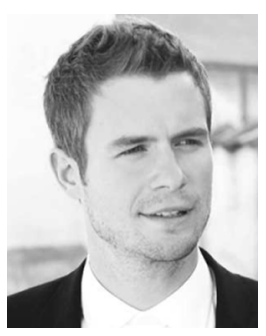

Daniel Georgiev received the B.Sc. degree in mechanical engineering, summa cum laude, from the University of New Mexico, Albuquerque, in 2002, and the M.Sc. degrees in mathematics and mechanical engineering and the Ph.D. degree in mechanical engineering from the University of Michigan, Ann Arbor, in 2005 and 2007, respectively.

Since then, he has served as a Research Associate in the SOS Lab and the School of Dentistry at the University of Washington, Seattle. In 2010, he joined the Department of Cybernetics at the University of West Bohemia, Pilsen, Czech Republic, as a visiting scholar, where he is currently conducting research on complex network estimation and biological system design.

Dr. Georgiev is a former student member of ASME and IEEE. He received a National Science Foundation Graduate Research Fellowship in 2002 and won the George Breece Prize for graduating first in his class at the University of New Mexico. 